\title{
A Quantum Chemistry Approach to Energies, Structures, and Spectroscopy of Doped Helium Clusters
}

\author{
R. Pérez de Tudela*, D. López-Durán*, M. P. de Lara-Castells*, R. Prosmiti*, O. \\ Roncero $^{*}$, G. Delgado-Barrio*, F. A. Gianturco ${ }^{\dagger}$, J. Jellinek ${ }^{* *}$ and P. Villarreal ${ }^{*}$ \\ *Instituto de Física Fundamental (CSIC), \\ Serrano 123, 28006 Madrid, Spain \\ ${ }^{\dagger}$ Department of Chemistry and INFM, The University of Rome, \\ Città Universitaria, 00185 Rome, Italy \\ ${ }^{* *}$ Chemical Sciences and Engineering Division, Argonne National Laboratory, \\ Argonne, Illinois 60439, USA
}

\begin{abstract}
Diffusion and path integral Monte Carlo methods are currently, and successfully, used to describe structures and binding energies of helium clusters doped with some impurity. For diatomic dopants, by considering the He atoms as "electrons" and the dopant as "nuclei" within a Hartree/Hartree-Fock framework, our group has developed a complementary tool which, in addition to the above mentioned properties, provides us with wave-functions. It allows to perform spectral simulations that can be compared with the experiment. This paper reviews the fundamental aspects of such quantumchemistry-like methodology in which, together with masses, Coulomb interactions are replaced by proper molecular ones. Extensions towards more accurate ab initio methodologies are outlined. Finally, the challenging scenario arising when the impurity is not immersed but attached to the nanodroplet, giving rise to an unusual "solar" system in which the planets no longer move around the dopant as a "sun", is addressed.

Keywords: electronic structure calculations, potential energy surface, weakly bounded clusters, He-nanodroplets, microscopic superfluidity PACS: $31.15 . \mathrm{A}, 31.15 . \mathrm{bw}, 31.15 . \mathrm{ve}, 31.15 . \mathrm{vj}, 31.50 . \mathrm{Bc}, 31.50 . \mathrm{Df}, 33.15 . \mathrm{Fm}, 33.20 . \mathrm{Vq}$
\end{abstract}

\section{INTRODUCTION}

Helium nanodroplets have proven to be a unique, gentle matrix in which to conduct high-resolution molecular spectroscopic experiments. Since the pioneering experiments on the infrared (IR) spectroscopy of $\mathrm{SF}_{6}$ embedded in helium droplets[1,2], further advances in the synthesis and characterization of solvated molecular species[3, 4] have allowed to carry out high resolution spectroscopic studies of different molecular species in cold environments. Actually, the droplets are evaporatively cooled to a very low temperature $(\leq 0.4 \mathrm{~K})$. However, they remain liquid even in the presence of a dopant, and are in fact superfluid[5], constituting a unique sample of the weakest existing solvent which can be loaded almost at will with atomic and molecular dopants. Thus, rotationally resolved IR spectra have been obtained for $\mathrm{Ar}_{n}-\mathrm{HF}[6], \mathrm{N}_{2}-\mathrm{HF}$ and OC-HF[7], and $\mathrm{C}_{2} \mathrm{H}_{4}$ [8] in helium nanodroplets. High-resolution IR measurements were also performed on $\mathrm{CO}[9,10]$ in small helium clusters. These studies revealed many interesting features closely related to the quantum nature of the solvent environment.

As a paradigm, infrared spectra of the carbonyl sulfide (OCS) molecule embedded in boson helium nanodroplets have provided direct evidence of the microscopic superfluid behavior of such an environment $[5,11]$. The IR spectrum of OCS inside a fermionic ${ }^{3} \mathrm{He}$-nanodroplet resembles the spectra of heavy molecules immersed in liquids and shows a broad, unstructured profile. In turn, the spectrum of OCS in a bosonic ${ }^{4} \mathrm{He}$-nanodroplet is quite similar to its isolated gas phase one presenting well-defined $\mathrm{P}$ and $\mathrm{R}$ branches, thus indicating that the molecule executes an essentially free rotation in the bosonic solvent.

\footnotetext{
${ }^{1}$ Author to whom correspondence should be addressed. E-mail: p.villarreal@imaff.cfmac.csic.es
}

\author{
International Conference of Computational Methods in Sciences and Engineering 2009 \\ AIP Conf. Proc. 1504, 240-253 (2012); doi: 10.1063/1.4771719 \\ (C) 2012 American Institute of Physics 978-0-7354-1122-7/\$30.00
}


Diverse quantum mechanical approaches have been used to theoretically describe the structure and energetics of both pure and doped ${ }^{4} \mathrm{He}$ boson clusters. Among them it is worth mentioning the Diffusion Monte Carlo technique (DMC) $[12,13,14]$, finite temperature Feymann's Path-Integral Monte Carlo (PIMC) methodology [15, 16, 17, 18, 19], as well as density functional theory approaches (see Ref. [20] for a review ). For fermionic clusters, the additional complexity involved by the nodal structure of the wavefunction introduces difficulties in applying these methodologies. For example, in DMC calculations, the nodes have to be envisaged a priori[21], and only recently a new technique for assessing the bias introduced in this way has been proposed[22].

Mimicking electronic structure calculations, an alternative quantum chemistry (QC) approach which considers the dopant molecule as nuclei and the the solvent atoms as electrons was firstly proposed and applied to study the lowest triplet state of $\mathrm{X}-\left({ }^{3} \mathrm{He}\right)_{2}$ clusters, with $\mathrm{X}=$ rare gas atom or $\mathrm{SF}_{6}[23]$. A similar approach, including a configuration interaction methodology, was used to study anthracene-He ${ }_{2}$ complexes[24, 25], including fermion and boson species, and variational calculations for rigid diatomic molecules surrounded by up to five boson He atoms have been also reported[26].

We have extended this idea to deal with larger $\mathrm{He}_{\mathrm{N}}-\mathrm{BC}(X)$ clusters, $\mathrm{N} \leq 60$, where $\mathrm{BC}$ is a conventional diatomic molecule at its ground electronic state[27, 28, 29, 30, 31, 32]. To consider the quantum nature of the surrounding helium atoms on the same footing, Hartree $(\mathrm{H})$ or Hartree-Fock (HF) methodologies have been implemented for bosons or fermions, respectively. The main difference between boson and fermion environments revealed through the calculations is that, in the latter case, it appears a high degree of degeneration of the different possible spin states at each given cluster size. For the case of mixed boson/fermion helium clusters, a self-consistent-field based procedure[33] that alternates between $\mathrm{H}$ and HF treatments was also successfully constructed[27, 28].

As usual, the electronic potential energy surface (PES) is a crucial issue in dealing with this kind of systems. Energies and structures of small clusters are expected to dramatically depend on the quality of the PES employed, in such a way that the use of $a b$ initio methodologies to obtain the corresponding PESs is highly desirable. In the case of $\mathrm{Br}_{2}$ as dopant, it has been shown[34] that a model PES for the tetra-atomic $\mathrm{He}_{2}-\mathrm{Br}_{2}$ system can be accurately constructed as the sum of two $\mathrm{He}-\mathrm{Br}_{2}$ triatomic potentials, analytically fitted to $a b$ initio points[35, 36] presenting minima at T-shaped and linear configurations, plus a semiempirical He-He pair interaction[37]. Similarly, ab initio calculations on the triatomic $\mathrm{He}-\mathrm{ICl}[38]$ predict three PES minima, which correspond to "linear" (He on the side of the I end of the $\mathrm{ICl}$ molecule), near T-shaped, and "anti-linear" ( $\mathrm{He}$ on the side of the $\mathrm{Cl}$ end of the $\mathrm{ICl}$ molecule) equilibrium structures. Modeling of the PES as a sum of the $\mathrm{He}-\mathrm{ICl}$ triatomic and $\mathrm{He}-\mathrm{He}$ pair interactions has been further verified by comparison with ab initio computations on $\mathrm{He}_{2}-\mathrm{ICl}[39]$. Hence, it is assumed that for $\mathrm{He}_{\mathrm{N}}-\mathrm{BC}$ clusters the corresponding PESs are in general well described as the sum of $\mathrm{N}$ triatomic He-BC potentials plus $\mathrm{N}(\mathrm{N}-1) / 2 \mathrm{He}-\mathrm{He}$ pair interactions.

One of the most appealing features of the QC approaches mentioned above is that they supply the wavefunction of the system thus allowing to perform spectral simulations which can be properly compared with the experiment. The IR absorption cross-sections for polar BC molecules and the intensities of the signal for the outgoing photon in vib-rotational Raman scattering for non-polar dopants can be obtained as a function of the incident photon energy. In either case, selection rules provide a clear distinction between bosonic or fermionic environments. In the former scenario, one gets that the allowed transitions are just the same that those corresponding to the isolated molecule, although the presence of the embedding helium atoms gives rise to some shift of the position of the lines. Also, as a consequence of possible relaxation processes, some broadening of the lines can be expected. On the other hand, in a fermion cluster, additional new transitions become allowed and contribute to the congestion of the corresponding spectrum. At low temperatures, such congestion is magnified due to the great degeneracy of spin states. Hence, well defined spectral profiles, similar to those in gas phase, can be envisaged for impurities in boson clusters, while broad unstructured profiles are conjectured for the corresponding spectra in fermion environments.

Alkali-metal atoms picked up by a beam of helium nanodroplets remain on the helium surface where they move around forming molecules in cold collisions. In this context, visible absorption spectra of cesium-doped cold helium nanodroplets involving the triplet ground state, ${ }^{3} \Sigma_{u}^{+}$, have been recently reported[40]. By itself, the cesium dimer in its triplet ground state plays an important role in cold atom- atom/molecule collisions, laser cooling of molecules, and coherent control[41]. Moreover, this electronic state has been selected with the ${ }^{1} \Sigma_{g}^{+}$ground state of $\mathrm{Cs}_{2}$ to experimentally detect extremely small variations in the electron-to-proton mass ratio[42], which is relevant to either verify or restrict quintessence models which try to explain the amount of dark energy that dominates the Universe. As a dopant, in the presence of helium clusters, the characteristics of the He-He and $\mathrm{He}-\mathrm{Cs}_{2}$ interactions[43] corroborate the above mentioned arrangement in which the impurity would be attached to, instead of immersed in, the cluster. A similar situation has been recently reported for $\mathrm{Li}_{2}$ doped ${ }^{4} \mathrm{He}$ clusters[44]. Thus, we wonder at what extent QC treatments are suitable to describe such a challenging scenario. 
In this paper we outline an overview of the QC methodology. The conceptual framework and theoretical models, including some advances reached in the implementation of ab initio methods which go beyond $\mathrm{H} / \mathrm{HF}$ approaches, and a description of the PIMC procedure, are reviewed in Section 2. The methodology to perform spectral simulations is described in Section 3, where outstanding results for $\mathrm{He}_{\mathrm{N}}-\mathrm{Br}_{2},-\mathrm{ICl}$ complexes are summarized. For clusters of ${ }^{4} \mathrm{He}$ with $\mathrm{Cs}_{2}\left({ }^{3} \Sigma_{u}^{+}\right)$, preliminary promising Hartree results together with PIMC predictions are included in Section 4. Finally, concluding remarks and an outlook of future work are given in Section 5.

\section{THEORETICAL FRAMEWORK AND MODELING}

\section{Quantum Chemistry Approach}

The Hamiltonian for a $\mathrm{He}_{N}-\mathrm{BC}$ system, using satellite coordinates $\left\{\left(\mathbf{r}, \mathbf{R}_{k}\right)\right\}$ where $\mathbf{r}$ is the vector joining the B and $\mathrm{C}$ atoms, and $\mathbf{R}_{k}$ are the vectors from the center of mass of the BC molecule to the different He atoms, can be written as

$$
H=H^{\mathrm{d}}+\sum_{k=1}^{N} H_{k}^{(\varepsilon)}+\sum_{k<l} \tilde{V}_{k l},
$$

which contains a diatomic part corresponding to the $\mathrm{BC}$ molecule

$$
H^{\mathrm{d}}=-\frac{\hbar^{2}}{2 m} \frac{\partial^{2}}{\partial r^{2}}+U(r)+\frac{\mathbf{j}^{2}}{2 m r^{2}},
$$

plus $N^{\varepsilon} \mathrm{He}-\mathrm{BC}$ triatomic Hamiltonians, $H_{k}^{(\varepsilon)},(\varepsilon=3$ or 4 for fermions or bosons, respectively), and the He-He interactions $\tilde{V}_{k l}$ including a potential term $V_{k l}$ and also a kinetic energy coupling which arises from the use of nonJacobi coordinates[45],

$$
\tilde{V}_{k l}=V_{k l}\left(\left|\mathbf{R}_{k}-\mathbf{R}_{l}\right|\right)-\frac{\hbar^{2}}{m_{B}+m_{C}} \nabla_{k} \cdot \nabla_{l}
$$

In Eq. (2), $\mathbf{j}$ is the angular momentum associated with $\mathbf{r}, m$ is the reduced mass of the diatomic molecule, and $U$ is the intramolecular diatomic potential. Triatomic Hamiltonians have the form

$$
H_{k}^{(\varepsilon)}=-\frac{\hbar^{2}}{2 \mu_{\varepsilon}} \frac{\partial^{2}}{\partial R_{k}^{2}}+\frac{\mathbf{l}_{k}^{2}}{2 \mu R_{k}^{2}}+W\left(R_{k}, r, \theta_{k}\right),
$$

where $\mu_{\varepsilon}$ is the reduced mass of the ${ }^{\varepsilon} \mathrm{He}-\mathrm{BC}$ system, $\mathbf{l}_{k}$ is the angular momentum associated with $\mathbf{R}_{k}$, and $W$ represents the atom-diatom intermolecular potential that depends on the pair of $\left(R_{k}, r\right)$ distances and the angle $\theta_{k}$ between the $\mathbf{R}_{k}$ and $\mathbf{r}$ vectors.

A body-fixed $(\mathrm{BF})$ coordinate system is chosen with the $Z^{\mathrm{BF}}$ axis along $\mathbf{r}$, and the quantum numbers associated with the projection of $\mathbf{L}=\sum_{k=1}^{N} \mathbf{l}_{k}$ and the total spin $\mathbf{S}$ on that axis are denoted by $\Lambda$ and $\Sigma$, respectively. We consider basis functions constructed as products of the form

$$
\mathscr{W}_{\Lambda S \Sigma v}^{J M}\left(\left\{\mathbf{R}_{k}\right\},\left\{\mathbf{s}_{k}\right\}, \mathbf{r}\right)=\mathscr{D}_{M \Omega}^{J^{*}}\left(\varphi_{r}, \theta_{r}, 0\right) \Psi_{\Lambda S v}^{J \Sigma}\left(\left\{\mathbf{R}_{k}\right\},\left\{\mathbf{s}_{k}\right\}, r\right),
$$

where $\mathbf{s}_{k}(k=1, \ldots, N)$ are the spin coordinates of the $N$ solvent atoms. In Eq. (5), the $\mathscr{D}_{M \Omega}^{J}$ functions are Wigner rotation matrices that depend on the polar components of $\mathbf{r}$ in the space-fixed (SF) frame, $J$ is the quantum number associated with the total angular momentum $\mathbf{J}=\mathbf{j}+\mathbf{L}+\mathbf{S}, M$ and $\Omega=\Lambda+\Sigma$ being quantum numbers associated with the projections of $\mathbf{J}$ on $Z^{\mathrm{SF}}$ and $Z^{\mathrm{BF}}$, respectively.

The quantum chemistry treatment takes the point of view of the dopant as being perturbed by the presence of the surrounding helium atoms. We resort to an adiabatic approach which completely neglects the diatomic Hamiltonian, i.e., considers BC as fixed "nuclei". Hence, the $\Psi$ function appearing in Eq. (5) is written as a product

$$
\Psi_{\Lambda S v}^{J \Sigma}\left(\left\{\mathbf{R}_{k}\right\},\left\{s_{k}\right\}, r\right)=\Phi_{\Lambda S}\left(\left\{\mathbf{R}_{k}\right\},\left\{\mathbf{s}_{k}\right\} ; r\right) \chi_{\Lambda S v}^{J \Sigma}(r)
$$


where the $\Phi$ function is solution, at fixed $r$ values, of

$$
\left[\sum_{k=1}^{N} H_{k}^{(\varepsilon)}\left(\mathbf{R}_{k} ; r\right)+\sum_{k<l}^{N} \tilde{V}_{k l}-E_{\Lambda S}(r)\right] \Phi_{\Lambda S}\left(\left\{\mathbf{R}_{k}\right\},\left\{\mathbf{s}_{k}\right\} ; r\right)=0 .
$$

Here, the $r$-dependent eigenvalues $E_{\Lambda S}$, which are degenerate with respect to $\Sigma$, modify the diatomic potential corresponding to the isolated BC molecule. Therefore the $\chi$ functions of Eq. (6) describe the vibrations of a distorted diatomic molecule

$$
\left[-\frac{\hbar^{2}}{2 m} \frac{\partial^{2}}{\partial r^{2}}+U(r)+E_{\Lambda S}(r)+\frac{\left\langle\mathbf{j}^{2}\right\rangle}{2 m r^{2}}-\varepsilon_{\Lambda S v}^{J \Sigma}\right] \chi_{\Lambda S v}^{J \Sigma}(r)=0
$$

where, neglecting Coriolis couplings, the averaged rotational term is approximated by[27, 28, 30]

$$
\left\langle\mathbf{j}^{2}\right\rangle \approx\left\langle\Phi_{\Lambda S}\left|\mathbf{L}^{2}\right| \Phi_{\Lambda S}\right\rangle+\hbar^{2}\left[J(J+1)+S(S+1)-2\left(\Lambda^{2}+\Sigma^{2}+\Lambda \Sigma\right)\right]
$$

with the $\mathbf{L}^{2}$ average being computed by using the distribution of $L$ values in the $\Phi_{\Lambda S}$ state[29].

The main assumptions involved in this approach (i.e., decoupling of orbital angular momentum from the BC rotation and adiabaticity of the $\mathrm{BC}$ stretching versus the He motions) have been verified on a system containing a heavy dopant as ${ }^{4} \mathrm{He}_{2}-\mathrm{Br}_{2}(\mathrm{X})[46]$. The conclusion of that work is that the adiabatic approximation is accurate in energies within 0.01 wavenumbers, while the angular momenta decoupling introduces errors one order of magnitude larger and can constitute the main source of discrepancies. Similar issues were recently addresed on the ${ }^{4} \mathrm{He}_{2}-\mathrm{N}_{2}(\mathrm{X})$ system[47], which contains a much lighter dopant. In line with the previous conclusions, adiabaticity maintains the same degree of accuracy but, owing to the lightness of the dopant, failure of angular momenta decoupling yields discrepancies of $\sim 2$ wavenumbers for rotation-less $J=0$ states, and is magnified with increasing the total angular momentum. However, at temperatures low enough, vib-rotational Raman simulations which use the $\mathrm{N}_{2}$ polarizabilities[48] reveal that the quantum chemistry treatment provides a very good description of the main features (position and intensity) of the corresponding lines[47].

\section{Hartree-Fock and Hartree methods}

The crucial point is to solve Eq. (7). In order to consider on the same footing fermionic or bosonic clusters we used Hartree-Fock or Hartree approaches, respectively, looking for the ground state of the system under study. For doped ${ }^{3} \mathrm{He}$ clusters $[27,28]$ the nuclear wave-function is written as an antisymmetrized product of one-fermion spin-orbitals, i.e. as a Slater determinant

$$
\Phi_{\Lambda S}=\frac{1}{\sqrt{N !}} \operatorname{det}\left[\phi_{1}\left(\mathbf{R}_{1}, \mathbf{s}_{1} ; r\right) \ldots \phi_{i}\left(\mathbf{R}_{i}, \mathbf{s}_{i} ; r\right) \ldots \phi_{N}\left(\mathbf{R}_{N}, \mathbf{s}_{N} ; r\right)\right]
$$

Each one-fermion wave function $\phi_{i}\left(\mathbf{R}_{i}, \mathbf{s}_{i} ; r\right)$ is a product of a spatial one-particle orbital $\psi_{i}\left(\mathbf{R}_{i} ; r\right)$ and a spin function $\sigma\left(\mathbf{s}_{i}\right)=\alpha\left(\mathbf{s}_{i}\right)$ or $\beta\left(\mathbf{s}_{i}\right)$. Depending on the spin-symmetry of the system, the spatial one-particle orbitals are found through the restricted closed-shell or open-shell Hartree-Fock methods.

In turn, the nuclear wave-function of doped ${ }^{4} \mathrm{He}$ clusters is written as a symmetrized Hartree product of singleparticle wave-functions[29, 30]. For a generic case, when $N_{i}$ bosons occupy the same single-particle orbital labeled as $i$, the $N$-boson total wavefunction is expressed as

$$
\Phi_{\Lambda 0}^{\left(N_{1}, \ldots, N_{M}\right)}=\frac{1}{\sqrt{\mathscr{N}}} \hat{\mathscr{S}}\left(\prod_{i=1}^{N_{1}} \psi_{1}\left(\mathbf{R}_{i} ; r\right) \prod_{j=N_{1}+1}^{N_{1}+N_{2}} \psi_{2}\left(\mathbf{R}_{j} ; r\right) \cdots \prod_{k=\left(N_{1}+\cdots+N_{M-1}\right)+1}^{N} \psi_{M}\left(\mathbf{R}_{k} ; r\right)\right)
$$

where $\sum_{i}^{M} N_{i}=N, M \leq N, \hat{\mathscr{S}}$ is the symmetrization operator, with $1 / \sqrt{\mathscr{N}}$ being a normalization factor. As already discussed[29], the energy can be written in terms of single particle energies of the bosons occupying the $\psi_{i}$ orbitals (denoted here as $\left.\varepsilon_{i}\right)$ and the equivalent to the Coulomb $\left(J_{i j}\right)$ and exchange $\left(K_{i j}\right)$ integrals appearing in electronic structure theory as

$$
E_{\left(N_{1}, \ldots, N_{M}\right)}^{(N)}=\sum_{i=1}^{M} N_{i} \varepsilon_{i}+\sum_{i, j=1}^{M} \frac{N_{i}\left(N_{j}-\delta_{i j}\right)}{2\left(1+\delta_{i j}\right)}\left(J_{i j}+K_{i j}\right),
$$


where the positive sign in front of the exchange integrals arises due to the symmetric nature of the bosonic wavefunction. In fact, for dopants immersed in boson helium clusters, it is found a Bose-like behavior, that is, the ground state corresponds to all the bosons occupying the same one-particle orbital,

$$
\Phi_{\Lambda 0}=\psi_{1}\left(\mathbf{R}_{1} ; r\right) \ldots \psi_{1}\left(\mathbf{R}_{i} ; r\right) \ldots \psi_{1}\left(\mathbf{R}_{N} ; r\right) .
$$

For bosons and singlet fermions, and starting from $N=2$, the initial orbitals are those corresponding to independent particles. Here on, the chosen initial orbitals were those obtained from the preceding calculation with $N-2$ particles. For $S \neq 0$ fermions, one starts with the optimized orbitals from the $S-1$ calculation for the same size cluster. In either case, spatial one-particle orbitals entering Eqs. (10)-(11) are expanded in terms of a finite basis set composed of products of radial and angular functions,

$$
\psi_{i}(\mathbf{R} ; r)=\sum_{n l m} c_{n l m}^{i} G_{n}(R ; r) Y_{l m}(\theta, \phi),
$$

where $Y_{l m}$ are spherical harmonics. Regarding radial basis functions one searches the ground level of a triatomic ${ }^{\varepsilon} \mathrm{He}-\mathrm{BC}$ subsystem at fixed orientations $\theta_{n}$,

$$
\left[-\frac{\hbar^{2}}{2 \mu_{\varepsilon}} \frac{\partial^{2}}{\partial R^{2}}+W\left(R, \theta_{n} ; r\right)-E_{n}(r)\right] g_{n}\left(R, \theta_{n} ; r\right)=0 .
$$

An orthonormal basis set of $G_{n}$ functions is then obtained from the $g_{n}$ functions through an orthogonalization Schmidt procedure.

In electronic structure calculations the electron-electron and electron-nucleus interactions are, respectively, repulsive and attractive. In contrast, our systems exhibit strong repulsion of both helium-helium and helium-dopant interactions at short distances. To avoid unphysically too large and positive energies [28, 49], one has to truncate the He-He potential at short distances $[28,29]$. This potential thus depends on a single parameter which is fitted for $N=2$ bosons and then used for all sizes, as well as for fermions. It is worth mentioning that convergence problems were found when applying the standard self-consistent method to iteratively solve the Fock equations. In order to force convergence to the global minimum it was found essential to use a direct minimization procedure proposed some years ago [50, 51].

To tackle mixed clusters containing $N_{\mathrm{f}}$ fermions and $N_{\mathrm{b}}$ bosons, one starts with a zero-order description corresponding to the pure case, i.e., one solves Eq. (7) separately for $\left(E_{\Lambda_{\mathrm{f}} S}, \Phi_{\Lambda_{\mathrm{f}} S}\right)$ and $\left(E_{\Lambda_{\mathrm{b}} 0}, \Phi_{\Lambda_{\mathrm{b} 0} 0}\right)$. One further resorts to a self-consistent-field (SCF) treatment[33] in which the wave function is expressed as a product $\Phi_{\Lambda_{\mathrm{f}} S} \Phi_{\Lambda_{\mathrm{b}} 0}$, and the factors are modified through iterative Hartree-Fock/ Hartree calculations up to convergence,

$$
\begin{aligned}
& {\left[\sum_{k=1}^{N_{\mathrm{f}}} H_{k}^{(3)}+\sum_{k<l}^{N_{\mathrm{f}}} \tilde{V}_{k l}+V_{\mathrm{f}}^{\left(N_{\mathrm{b}}\right)}-\tilde{E}_{\Lambda_{\mathrm{f}} S}(r)\right] \Phi_{\Lambda_{\mathrm{f}} S}=0} \\
& {\left[\sum_{k=1}^{N_{\mathrm{b}}} H_{k}^{(4)}+\sum_{k<l}^{N_{\mathrm{b}}} \tilde{V}_{k l}+V_{\mathrm{b}}^{\left(N_{\mathrm{f}}\right)}-\tilde{E}_{\Lambda_{\mathrm{b}} 0}(r)\right] \Phi_{\Lambda_{\mathrm{b}} 0}=0,}
\end{aligned}
$$

where $V_{\mathrm{f}}^{\left(N_{\mathrm{b}}\right)}$ and $V_{\mathrm{b}}^{\left(N_{\mathrm{f}}\right)}$ stand for the averages $\left\langle\Phi_{\Lambda_{\mathrm{b}} 0}\left|V_{\mathrm{fb}}\right| \Phi_{\Lambda_{\mathrm{b}} 0}\right\rangle$ and $\left\langle\Phi_{\Lambda_{\mathrm{f}} S}\left|V_{\mathrm{fb}}\right| \Phi_{\Lambda_{\mathrm{f}} S}\right\rangle$, respectively.

The effective diatomic Hamiltonian is estimated through replacement of $E_{\Lambda_{\mathrm{f}}, S}$ in Eq. (8) with a sum of the separate energies plus the first order perturbation term coming from fermion-boson interactions,

$$
V_{\mathrm{fb}}=\sum_{k=1}^{N_{\mathrm{f}}} \sum_{l=1}^{N_{\mathrm{b}}} \tilde{V}_{k l}
$$

i.e., one writes

$$
E_{\Lambda_{\mathrm{fb}} S}(r)=E_{\Lambda_{\mathrm{f}} S}(r)+E_{\Lambda_{\mathrm{b}} 0}(r)+\overline{\bar{V}}_{\mathrm{fb}}
$$

where $\overline{\bar{V}}_{\mathrm{fb}}=\left\langle\Phi_{\Lambda_{\mathrm{f}} S} \Phi_{\Lambda_{\mathrm{b}} 0}\left|V_{\mathrm{fb}}\right| \Phi_{\Lambda_{\mathrm{b}} 0} \Phi_{\Lambda_{\mathrm{f}} S}\right\rangle$. In Eq. (18), $\Lambda_{\mathrm{fb}}=\Lambda_{\mathrm{f}}+\Lambda_{\mathrm{b}}$. Indeed, since $\mathbf{L}=\sum_{k=1}^{N_{\mathrm{f}}} \mathbf{l}_{k}+\sum_{j=1}^{N_{\mathrm{b}}} \mathbf{l}_{j}$, one has to calculate again $\left\langle\mathbf{L}^{2}\right\rangle$. 


\section{Beyond the Hartree/ Hartree-Fock approaches}

To include explicitly the He-He correlation and to obtain excited states is necessary to go beyond H/HF methodologies. Thus, an efficient full configuration-interaction (FCI) treatment[52] has been recently developed and applied to ${ }^{3} \mathrm{He}_{N}-\mathrm{Br}_{2}$ systems, $N \leq 4$, from which "exact" results, within the adiabatic approximation and a given basis set, can be obtained. One crucial point in the development of this treatment was to incorporate the Jacobi-Davidson (JD) modification[53] of the standard Davidson algorithm [54]. In fact, by using the pure Davidson method, which in quantum-chemistry calculations is nearly always used, it was not possible to obtain converged results whereas the JD procedure gives a very fast convergence. The very poor performance of the Davidson method stems, again, from the very strong repulsion of both $\mathrm{He}-\mathrm{He}$ and $\mathrm{He}-\mathrm{BC}$ interactions, which gives rise to a Hamiltonian matrix with very large off-diagonal elements. In the JD method, one approximately solves the Newton-Raphson correction equation iteratively before each Davidson macro iteration. This treatment has also been successfully applied to ${ }^{3} \mathrm{He}_{N^{-}}$ $\mathrm{Cl}_{2}$ systems[55], and additional methodological and computational details of its implementation have been recently provided[56]. Converged results are used to analyze global energetic and structural aspects, and also the configuration makeup of the wave functions, associated with the ground and low-lying "solvent" excited states. The study reveals that, besides the fermionic nature, key roles in determining total binding energies and wave-function structures are played by the strong repulsive core of the He-He potential as well as its very weak attractive region. The most stable arrangement somehow departs from that of $N$ He atoms equally spaced on an equatorial ring around the dopant. The present results [56] for $N=4$ fermions indicate the structural pairing of two ${ }^{3} \mathrm{He}$ atoms at opposite sides on a broad belt around the dopant, executing a sort of asymmetric umbrella motion. This pairing is a compromise between maximizing the He-He and the He-dopant attractions, and suppressing at the same time the hard-core repulsion[56]. Indeed FCI results corroborate the main HF finding as regards the high degeneracy of ground states of different multiplicity $[52,56]$.

\section{Path Integral Monte Carlo approach}

The PIMC approach employed here has been described elsewhere $[15,16]$. Briefly, the density matrix at a temperature $T$ is replaced by the product of $M$ density matrices at higher temperatures $M \times T$ :

$$
\rho\left(\mathscr{R}_{1}, \mathscr{R}_{M+1} ; \beta\right)=\int d \mathscr{R}_{2} \ldots d \mathscr{R}_{M} \prod_{\alpha=1}^{M} \rho\left(\mathscr{R}_{\alpha}, \mathscr{R}_{\alpha+1} ; \tau\right),
$$

where $\beta=1 / k_{B} T, k_{B}$ is the Boltzmann constant, and $\tau=\beta / M . \mathscr{R}_{\alpha}$ is the vector which collects the cartesian positions of the $N+2$ particles: $\mathscr{R}_{\alpha} \equiv\left\{\mathbf{r}_{1}^{\alpha}, \ldots, \mathbf{r}_{N+2}^{\alpha}\right\}$, being $\mathbf{r}_{i}^{\alpha}$ the position vector of the $i$-th atom at the time slice or imaginary time $\alpha$. It is important [15] to distinguish between $\tau$ and the iteration step, intrinsic to the movements of the paths in the Monte Carlo (MC) calculation. The $M$ number defines the beads which constitute each polymer or quantum path [15]. The specific case $M=1$ corresponds to the classical description of the system [57]. The energy can be obtained as the thermal average of the Hamiltonian $H$ as:

$$
\langle E(T)\rangle=Z^{-1} \int d \mathscr{R}_{1} \int d \mathscr{R}_{M+1}\left\langle\mathscr{R}_{M+1}|\hat{H}| \mathscr{R}_{1}\right\rangle \rho\left(\mathscr{R}_{1}, \mathscr{R}_{M+1} ; \beta\right)
$$

where $Z=\int d \mathscr{R} \rho(\mathscr{R}, \mathscr{R} ; \beta)$ is the partition function. Eq. (20) can be expressed, by using the energy estimators proposed in Refs. [58, 59] as:

$$
\langle E(T)\rangle=\left\langle\frac{3 N}{2} k_{B} T-\frac{1}{2 M} \sum_{\alpha=1}^{M} \sum_{i=1}^{N+2}\left(\mathbf{r}_{i}^{\alpha}-\mathbf{r}_{i}^{M}\right) \cdot \mathbf{F}_{i}^{\alpha}+\frac{1}{M} \sum_{\alpha=1}^{M} \sum_{i<j}^{N+2} V\left(\mathrm{r}_{i j}^{\alpha}\right)\right\rangle,
$$

where $\mathbf{r}_{i}^{M}=M^{-1} \sum_{\alpha=1}^{M} \mathbf{r}_{i}^{\alpha}$ and $\mathbf{r}_{i j}^{\alpha}=\left|\mathbf{r}_{i}^{\alpha}-\mathbf{r}_{j}^{\alpha}\right|$. The first term in Eq. (21) corresponds to the classical kinetic energy where the degrees of freedom from the center-of-mass (CM) of the whole system have been subtracted. The second one is a quantum correction where $\mathbf{F}_{i}^{\alpha}=-\sum_{j \neq i}^{3} \nabla_{\mathbf{r}_{i}^{\alpha}} V\left(\mathrm{r}_{i j}^{\alpha}\right)$ is the force experienced by the $i$-particle on the $\alpha$ slice and the third term describes the interaction between each pair of particles on that $\alpha$ slice. Note that the force $\mathbf{F}_{i}^{\alpha}$ is straightforwardly estimated as long as the PES is analytically described. Finally, the integration is carried out via a Metropolis MC algorithm[60], as an average over a number of cyclic paths $\left\{\mathscr{R}_{1}, \mathscr{R}_{2}, \ldots, \mathscr{R}_{M}, \mathscr{R}_{M+1}=\mathscr{R}_{1}\right\}$ sampled according to a probability density proportional to the factorised product of $M$ density matrices of Eq. (19). The present 
study also includes the analysis of diverse two-particle properties as, eg. the angular distribution of $\cos \gamma, \gamma$ being the angle formed by whatever be the couple of vectors $\mathbf{R}_{k}, \mathbf{R}_{l}(k \neq l)$ joining the corresponding He atoms and the center of mass of the cesium dimer. It is calculated as:

$$
D(\cos \gamma)=\frac{1}{N(N-1) M} \sum_{\alpha=1}^{M} \sum_{k<l}^{N} \delta\left(\cos \gamma-\hat{\mathbf{R}}_{k}^{\alpha} \cdot \hat{\mathbf{R}}_{l}^{\alpha}\right), \quad \text { where } \hat{\mathbf{R}}_{k}^{\alpha}=\mathbf{R}_{k}^{\alpha} / \mathbf{R}_{k}^{\alpha}
$$

\section{SPECTRAL SIMULATIONS}

\section{Vib-rotational Raman Spectra}

For homonuclear dopants, $\mathrm{C}=\mathrm{B}$, we consider a scattering process where a photon of energy $\hbar \omega_{0}$ collides with the $\mathrm{B}_{2}$ $\mathrm{He}_{N}$ doped cluster in an initial state $|i\rangle$ inducing on the diatomic polarizable system an electric dipole which, in turn, may emit a photon of energy $\hbar \omega_{f i}$ while the system emerges in a final state $|f\rangle$. We assume linearly polarized incident light with its electric vector defining the SF $Z$ direction and that propagates along the $Y$ axis (while the scattered light is detected along the $X$ axis). Making use of the diatomic polarizability, which is supposed to be unaffected by complexation, in the BF frame, the components of the induced dipole in SF become[27, 28, 32]

$$
\mu_{k} \sim \sum_{m=-1}^{+1}(-1)^{m} \alpha_{m} \sum_{n=0}^{2}(2 n+1)\left(\begin{array}{ccc}
1 & 1 & n \\
-m & m & 0
\end{array}\right)\left(\begin{array}{ccc}
1 & 1 & n \\
-k & 0 & k
\end{array}\right) \mathscr{D}_{0-k}^{n}\left(\varphi_{r}, \theta_{r}, 0\right)
$$

The matrix elements of the induced dipole moment components between initial and final states of type (5), $\mu_{k}^{f, i}=$ $\left\langle\mathscr{W}_{f}\left|\mu_{k}\right| \mathscr{W}_{i}\right\rangle$, can be estimated after carrying out an integral over three Wigner matrices as

$$
\mu_{k}^{f, i} \sim \delta_{\Lambda_{f} \Lambda_{i}} \delta_{S_{f} S_{i}}(-1)^{M_{i}} \sum_{m, n}(-1)^{m} \alpha_{m}^{f, i}(2 n+1)\left(\begin{array}{ccc}
1 & 1 & n \\
-m & m & 0
\end{array}\right)\left(\begin{array}{ccc}
1 & 1 & n \\
-k & 0 & k
\end{array}\right)\left(\begin{array}{ccc}
J_{i} & n & J_{f} \\
-M_{i} & 0 & M_{i}
\end{array}\right)\left(\begin{array}{ccc}
J_{i} & n & J_{f} \\
-\Omega_{i} & -k & \Omega_{f}
\end{array}\right)
$$

where $\alpha_{m}^{f, i}=\left\langle\chi_{\Lambda_{i} S_{i} v_{f}}^{J_{f} \Sigma_{i}}\left|\alpha_{m}\right| \chi_{\Lambda_{i} S_{i} v_{i}}^{J_{i} \Sigma_{i}}\right\rangle$. Note that in the boson scenario $\left(\Lambda_{i}=\Omega_{i}=\Omega_{f}=0\right)$ only $\mu_{0}$ survives, and the scattered light should emerge polarized along $Z$. In this case, the sum over $n$ runs only over the values 0 and 2 , giving rise to standard diatomic-like selection rules $\Delta J=0, \pm 2$ thus contributing to $\mathrm{O}, \mathrm{Q}$, and $\mathrm{S}$ branches in the spectrum. To stress the difference of boson/fermion frameworks, we consider detection along the $X$ axis for linear, parallel polarization. In terms of the spherical $\alpha_{f, i}=\left(\alpha_{0}^{f, i}+2 \alpha_{1}^{f, i}\right) / 3$, and the anisotropic $\beta_{f, i}=\left(\alpha_{0}^{f, i}-\alpha_{1}^{f, i}\right)$ parts of the polarizability[61], one finally finds[27, 28, 32]

$$
\mu_{0}^{f, i} \sim \alpha_{f, i}\left(\begin{array}{ccc}
J_{i} & 0 & J_{f} \\
-M_{i} & 0 & M_{i}
\end{array}\right)\left(\begin{array}{ccc}
J_{i} & 0 & J_{f} \\
-\Omega_{i} & 0 & \Omega_{f}
\end{array}\right)+\frac{2 \beta_{f, i}}{3}\left(\begin{array}{ccc}
J_{i} & 2 & J_{f} \\
-M_{i} & 0 & M_{i}
\end{array}\right)\left(\begin{array}{ccc}
J_{i} & 2 & J_{f} \\
-\Omega_{i} & 0 & \Omega_{f}
\end{array}\right) .
$$

The third component of $\mathrm{J}$ is conserved in $\mathrm{SF}(\Delta M=0)$ and also in $\mathrm{BF}(\Delta \Omega=0)$ frames. As compared with bosons' spectra, note that remarkable changes appear in the spectra of complexes containing fermions with $\Omega_{i}=\Omega_{f} \neq 0$. In fact, transitions with $\Delta J= \pm 1$ become allowed and, thus, $\mathrm{P}$ and $\mathrm{R}$ branches (with the exception of $\mathrm{P}(1)$ and $\mathrm{R}(0)$ ) should appear in the spectrum.

For a fixed energy of the incident photon we introduce a Boltzmann distribution over cluster states at a given temperature $T$ and average over initial rotational states. Hence, a line of intensity

$$
I_{f i}(T) \propto \frac{e^{-\left(\varepsilon_{i} / k T\right)}}{\sum_{i} e^{-\left(\varepsilon_{i} / k T\right)}} \frac{1}{2 J_{i}+1} \sum_{M_{i}}\left|\mu_{0}^{f, i}\right|^{2}
$$

would appear at an energy $\hbar \omega_{f i}=\hbar \omega_{0}-\left(\varepsilon_{\Lambda_{i} S_{i} v_{f}}^{J_{f} \Sigma_{i}}-\varepsilon_{\Lambda_{i} S_{i} v_{i}}^{J_{i} \Sigma_{i}}\right)$ of the scattered photon. In Eq. (26), $\varepsilon_{i}$ means $\varepsilon_{\Lambda_{i} S_{i} v_{i}}^{J_{i} \Sigma_{i}}$.

\section{Infrared Spectra}

By absorption of one photon, an heteronuclear molecule BC possessing a permanent dipole moment can be promoted from an initial vib-rotational level $|i\rangle$ to a final vib-rotational one $|f\rangle$ within the same electronic state. 
The energy $\hbar \omega_{i f}$ of the absorbed photon matches the energy difference between the initial and final states, belonging to the infrared region. When such a molecule is embedded in a bath of He atoms, one considers a similar process,

$$
\mathrm{He}_{N}-\mathrm{BC}(i)+\hbar \omega_{i f} \rightarrow \mathrm{He}_{N}-\mathrm{BC}(f),
$$

where now the indices $i$ and $f$ refer, respectively, to the initial and the final states of the entire $\mathrm{He}_{N}-\mathrm{BC}$ cluster. In the electric dipole approximation within the first order perturbation theory, the absorption intensity is proportional to the square modulus of the matrix element of the transition moment operator, $\boldsymbol{\mu} \cdot \hat{\boldsymbol{e}}$, computed using the functions defined by Eq. (5); here, $\boldsymbol{\mu}$ is the dipole moment of the solvated molecule, and $\hat{\boldsymbol{e}}$ is the polarization of the electric field. The electric field defines a natural SF frame, while usually the dipole moment is expressed in a BF reference frame. Therefore, a rotation should be performed to express the dipole moment in the SF frame, i.e., $\boldsymbol{\mu}^{S F}=\boldsymbol{R}^{-1} \boldsymbol{\mu}^{B F}$. As ab initio calculations on ICl reveal[31], the dipole moment of the solvated molecule is only weakly affected by the interactions with the surrounding He atoms and its direction remains along the diatomic bond. By considering again linearly polarized light, its direction of polarization defining the SF Z-axis, one obtains[30, 31]

$$
\left\langle\mathscr{W}_{i}|\boldsymbol{\mu} \cdot \hat{\boldsymbol{e}}| \mathscr{W}_{f}\right\rangle \propto(-1)^{M_{i}}\left\langle\chi_{f}\left|\mu_{0}\right| \chi_{i}\right\rangle \delta_{\Lambda_{f} \Lambda_{i}}\left(\begin{array}{ccc}
J_{i} & 1 & J_{f} \\
-M_{i} & 0 & M_{i}
\end{array}\right)\left(\begin{array}{ccc}
J_{i} & 1 & J_{f} \\
-\Omega_{i} & 0 & \Omega_{i}
\end{array}\right) .
$$

Since $\Omega=\Lambda+\Sigma$, for $\Lambda=0(\Sigma)$ states of bosonic clusters, the second $3-j$ symbol in Eq. (28) vanishes unless $\Delta J= \pm 1$. Hence, in a bosonic scenario, only P and R branches can appear in the spectrum, where Q transitions are forbidden. In turn, when $\Omega \neq 0$ states are involved (e.g. because of the presence of fermionic components in the solvent and/or the presence of $\Pi, \Delta, .$. states) Q transitions become also allowed. Once again, a Boltzmann distribution of rotational states of the clusters at a given temperature $T$ gives rise to a line of intensity

$$
I_{f i}(T) \propto \frac{e^{-\left(\varepsilon_{i} / k T\right)}}{\sum_{i} e^{-\left(\varepsilon_{i} / k T\right)}} \frac{1}{2 J_{i}+1} \sum_{M_{i}}\left|\left\langle\chi_{f}\left|\mu_{0}\right| \chi_{i}\right\rangle\left(\begin{array}{ccc}
J_{i} & 1 & J_{f} \\
-M_{i} & 0 & M_{i}
\end{array}\right)\left(\begin{array}{ccc}
J_{i} & 1 & J_{f} \\
-\Omega_{i} & 0 & \Omega_{i}
\end{array}\right)\right|^{2}
$$

at an energy $\hbar \omega_{f i}=\left(\varepsilon_{\Lambda_{i} S_{i} v_{f}}^{J_{f} \Sigma_{i}}-\varepsilon_{\Lambda_{i} S_{i} v_{i}}^{J_{i} \Sigma_{i}}\right)$.

\section{Continuum profiles}

Several relaxation processes can contribute to the broadening of the spectral lines. We assume that the diatomic impurity, as a consequence of its interaction with the radiation, reaches a vibrationally excited state. The excess of energy can eventually flow from the dopant to some of the weak bonds causing its breaking, that is, a direct vibrational predissociation (VP) process. In spite of VP competes with other relaxation mechanisms, as e.g. intramolecular energy redistribution, and evaporative cooling[62], we extend previous studies on three-atomic[63] and tetra-atomic[64, 65, 66] species to larger clusters to get a rough estimation of the VP width[27, 28, 30, 32]. In the framework of an adiabatic angular model[67] one first estimates the $\theta$-dependent VP width for the ${ }^{\varepsilon} \mathrm{He}-\mathrm{BC}\left(X, v_{f}\right)$ triatomic species, $\gamma_{f}$. Then, by integrating over all variables except one the square modulus of the function $\Phi_{\Lambda S}\left(\left\{\mathbf{R}_{k}\right\},\left\{\mathbf{s}_{k}\right\} ; r_{e q}\right)$, defined by Eq. (7), the angular distribution $\mathscr{D}_{\Lambda S}^{(N)}(\theta)$ of helium atoms around the dopant is generated. It is normalized to the cluster size $N$. An average of the triatomic VP width using the precedent angular distribution leads to a global VP width

$$
\Gamma_{f}^{(N)}=\int_{0}^{\pi} d \theta \mathscr{D}_{\Lambda S}^{(N)}(\theta) \gamma_{f}(\theta)
$$

For mixed clusters containing $N_{f} / N_{b}$ fermion/boson helium atoms, the VP width is simply estimated as a mean value $\Gamma_{f}^{\left(N_{f}+N_{b}\right)}=\left(N_{f} \Gamma_{f}^{\left(N_{f}\right)}+N_{b} \Gamma_{f}^{\left(N_{b}\right)}\right) /\left(N_{f}+N_{b}\right)$. Finally, by dressing with Lorentzian functions the stick lines of different intensities, and summing over all transitions, a continuum profile for vib-rotational Raman scattering signal or IR photo-absorption cross-section is obtained:

$$
\sigma_{N}(\omega ; T)=\frac{1}{2 \pi} \sum_{f, i} \frac{\Gamma_{f}^{(N)}}{\hbar^{2}\left(\omega-\omega_{f i}\right)^{2}+\left(\Gamma_{f}^{(N)} / 2\right)^{2}} I_{f i}(T) .
$$




\section{$\mathrm{Br}_{2}$ and ICl doped clusters: Accurate results}

The main energetic and structural properties of $\mathrm{He}_{N}-\mathrm{Br}_{2}$ clusters, analyzed through the QC approach, have been already described[27, 28, 29]. For boson solvents, the $H$ approach was tested[29] by comparing with results obtained through the "exact" DMC treatment[14]. In turn, H-F results[28] and FCI ones[52] were compared in Ref. ([32]) for the fermion environment. Assuming a vibrational excitation $v=1 \leftarrow 0 \mathrm{of} \mathrm{Br}_{2}$, the dependence of the corresponding vib-rotational Raman spectra on the statistics of the surrounding helium atoms were clearly shown in Ref. ([32]). The simulations were carried out at a temperature of $T=2 \mathrm{~K}$, which is below the transition temperature to superfluidity for ${ }^{4} \mathrm{He}\left(T_{\lambda}=2.12 \mathrm{~K}\right)$, but over the corresponding one for ${ }^{3} \mathrm{He}\left(T_{\lambda}=3 \times 10^{-3} \mathrm{~K}\right)$ [5]. The properties of the impurity were assumed to be unaffected by complexation, and the necessary polarizabilities were taken from the literature[68]. Focusing on the region of the $S(1)$ branch, we have depicted through figures 6a-6d[32] the gradual evolution of the spectra in terms of the relative content of fermion/boson species solvating the impurity. These figures display continuum profiles of the intensity of the outgoing photon as a function of its energy (relative to the energy of the incoming photon which has been chosen to match the vibrational excitation of the bare diatomic molecule, i.e., $\hbar \omega_{0}=323.15616365 \mathrm{~cm}^{-1}$ ). They correspond to bromine doped clusters containing: (a) $18{ }^{4} \mathrm{He}$ atoms, (b) $18{ }^{3} \mathrm{He}$ atoms, (c) a mixture of $18{ }^{3} \mathrm{He}$ fermions plus $6{ }^{4} \mathrm{He}$ bosons, and finally (d) a mixture of $18{ }^{3} \mathrm{He}$ fermions plus 18 ${ }^{4} \mathrm{He}$ atoms. The main lines contributing to the different profiles are specified as $\mathrm{B}\left(J_{S|\Sigma|}\right)$, where $\mathrm{B}=\mathrm{S}$ or $\mathrm{R}$ means the line, $J=J_{i}$, and an additional superscript on the total angular momentum, $J^{-}$, specifies that the spin projection $\Sigma$ has the opposite sign to $\Lambda$. Figure 6 a of Ref. ([32]) shows the typical look corresponding to a bosonic cluster, i.e. a pure Lorentzian profile coming from a unique Stokes line. As opposite, Figure 6b[32] displays a rather complicated profile since there are several S contributing lines coming from near degenerated spin multiplets. Moreover, R lines (allowed in this scenario) do appear interplaced among $\mathrm{S}$ lines and contribute to increase the congestion of the spectrum. The same relevant $S$ lines $(S=3$ and 4$)$ are present in the spectrum of the cluster which incorporates six boson helium atoms to the previuos cluster, Figure 6c[32], where a rather high degree of congestion remains. However, as compared with the precedent case, those $\mathrm{S}$ lines are closer and almost hide the presence of a unique noticeable $\mathrm{R}$ line. Finally, in figure 6d[32], and apart from the small contribution of $\mathrm{R}$ lines on the tail to the red, it is clear that the only important contribution comes from a $S=3$ spin. As a consequence, an almost Lorentzian profile is recovered. This sequence constitutes a picture completely similar to the reported experiments on OCS in nanodroplets[5, 11, 69], and can be explained as a mass effect: as long as boson helium atoms are added to the fermionic cluster, they reach spatial regions closer to the dopant than the fermion atoms, and even eject them to outer regions.

The main differences exhibited by $\mathrm{ICl}$ containing helium clusters, as compared with those doped with $\mathrm{Br}_{2}$, reside on the polar character of the dopant, allowing to simulate IR spectra which are more closely related to the experiments reported for linear dopants as OCS[5, 11]. As already mentioned, each triatomic potential He-ICl exhibits three minima which, in decreasing order of binding energy, go from a linear He-I-Cl, through a near T-shaped, to an antilinear HeCl-I configuration[38]. Hence, the PESs described as the sum of triatomic potentials plus the He-He interactions show a higher degree of anisotropy than those of clusters doped with $\mathrm{Br}_{2}$. Such an anisotropy has persistent effects in clusters of up to $30{ }^{4} \mathrm{He}$ atoms[30]. By means of $\mathrm{H}$ calculations, binding energies, density distributions, IR spectra, and rotational constants leading to effective inertia moments, have been already reported for ${ }^{4} \mathrm{He}_{N}$-ICl bosonic clusters with $N \leq 30$ [30]. Similarly, H-F calculations were performed for all the spin states of the ${ }^{3} \mathrm{He}_{18}-\mathrm{ICl}(\mathrm{X})$ fermionic cluster[31]. The results were summarized in Table 1 of that reference. The states correspond to " $\Sigma$ " states $(\Lambda=0)$ for $S=0,3-6$ and 8 and to " $\Pi$ " states $(\Lambda=1)$ for $S=1,2,7$ and 9 . $\mathbf{L}^{2}$ averages, obtained after getting the corresponding L-distributions[29], become larger than that corresponding to the bosonic case by a factor of 2-3. The binding energies follow a logarithmic dependence on the ICl bond length $r$ in the neighborhood of the equilibrium value $(E \simeq A \log (\tilde{r}-2)+B, \tilde{r}=r / \AA)$. The small variation of the slope $A$ parameter with the spin values up to $S=5$, and of the binding energy at $r=3.0 \AA B$, along all the $S$ values, reflects a high degree of degeneration, a feature always exhibited by fermionic clusters.

IR simulations were performed by considering an excitation that promotes the ICl dopant from the $v=0$ ground vibrational state to the $v=1$ excited one[30,32,31]. Ab initio calculations at the CCSD(T) level of the theory had shown that the dipole moment $\boldsymbol{\mu}$ of the ICl molecule in clusters containing one or two helium atoms is only slightly affected by their presence, remaining very close to that of the isolated molecule, showing a linear dependence on the interdiatomic bond-length[30]. At a temperature of $0.5 \mathrm{~K}$, figure 8a in Ref. ([32]) depicts the IR spectrum for a boson cluster containing eighteen helium atoms, i.e. the absorption cross-section as a function of the energy of incident photon (relative to the $v=0 \leftarrow 1$ transition of the bare ICl molecule, i.e., $\hbar \omega_{01}=380.084399 \mathrm{~cm}^{-1}$ ). A VP width of $4.5 \times 10^{-3} \mathrm{~cm}^{-1}$ was considered to dressing stick lines[30]. To simulate the spectrum of this molecule within a boson 
nanodroplet, the stick spectrum of the cluster containing thirty boson atoms was dressed with the extrapolated VP width $(N \rightarrow \infty)$ of $8.2 \times 10^{-3} \mathrm{~cm}^{-1}$ [31]. The resulting profile, shown in figure 2 of Ref. ([31]), is completely similar to the precedent one with the exception of a very small blue-shift and a slightly larger broadening of the different lines. As predicted by the selection rules, Q branches are absent in these simulations since just " $\Sigma$ " states are involved for bosonic environments. This completely agrees with the experimental findings on OCS in nanodroplets (see, e.g., the upper panel of Fig. 1 at Ref. [5]). The dominant branch is $\mathrm{R}(0)$, followed by $\mathrm{P}(1)$, R(1), etc., and only one line contributes to each branch. In turn, the IR spectrum of ICl within a cluster of eighteen fermion helium atoms, figure 3 of Ref. ([31]), showed marked differences. It was simulated even at a lower temperature of $0.3 \mathrm{~K}$, the VP widths for the different spin states ranging from $10.5 \times 10^{-3} \mathrm{~cm}^{-1}(S=5)$ to $14.5 \times 10^{-3} \mathrm{~cm}^{-1}(S=0)[32]$. The R(0) branch remained as dominant, but Q branches appeared as the second in importance, and all the branches stemmed from several transitions as a consequence of the quasi-degeneration in energy of different spin states. To simulate the spectrum of $\mathrm{ICl}$ in a fermion nanodroplet, additional $\mathrm{H}-\mathrm{F}$ calculations for the singlet spin state of clusters containing up to thirty fermion helium atoms were carried out[31]. Although increasing, the VP widths did not exhibit a smooth behavior as a function of the cluster size, but abrupt jumps were obtained for this magnitude at $N=10,18$, and more markedly, 30. Hence, it was not possible to perform a proper extrapolation of this magnitude for $N \rightarrow \infty$ but a sort of enveloping of the profile of Figure 3[31] was conjectured by considering VP widths fifteen times larger than the original ones. Such an envelope was shown in Figure 4 of Ref. ([31]). As can be realized, it exhibits a great similarity with the experiment on OCS in a fermion nanodroplet reported in the lower panel of Fig. 1 at Ref. [5]. It can be assumed that the most prominent peak is reminiscent of the $\mathrm{R}(0)$ branch, while the clear shoulder located to the red mainly stems from the contribution of Q branches.

\section{CESIUM DIMER DOPING HELIUM CLUSTERS}

\section{The Potential Energy Surface}

Theoretical calculations on the cesium dimer indicate that the ${ }^{3} \Sigma_{u}^{+}$state has a shallow well[70]. Recently, up to 41 vibrational levels have been experimentally detected and, based on these data, an analytical potential curve has been derived[71]. Regarding the $\mathrm{He}-\mathrm{Cs}_{2}\left({ }^{3} \Sigma_{u}^{+}\right)$complex, ab initio calculations using the spin restricted single and double excitations coupled cluster method with perturbative triples $(\operatorname{RCCSD}(T))$, correlating only valence electrons, have been recently performed[43]. To this end, the CRENBL[72] effective core potential and a basis set d-aug-cc-pVQZ were found to fulfill the compromise between accuracy and computational time. Frozing the cesium at its equilibrium bond-length, the calculations were carried out for several distances between the center of mass of the dimer and the helium atom at ten orientations in the range $0 \leq \theta \leq \pi / 2$ up to a total of 355 points. The surface presents the global minimum at the perpendicular orientation, where there is a shallow well with a depth of $\sim 2 \mathrm{~cm}^{-1}$ placed at $R \sim 6.75 \AA$. That depth gradually decreases to $\sim 0.75 \mathrm{~cm}^{-1}$, while $R$ increases to $\sim 11.5 \AA$, at linear arrangements. A simple model of adding atom-atom Lennard-Jones potentials, with well-depth and equilibrium distance parameters depending on the angular orientation, was found to accurately reproduce the ab initio points for interaction energies $\leq 5 \mathrm{~cm}^{-1}$ [43]. Using this analytical form, variational calculations at zero total angular momentum predict a single

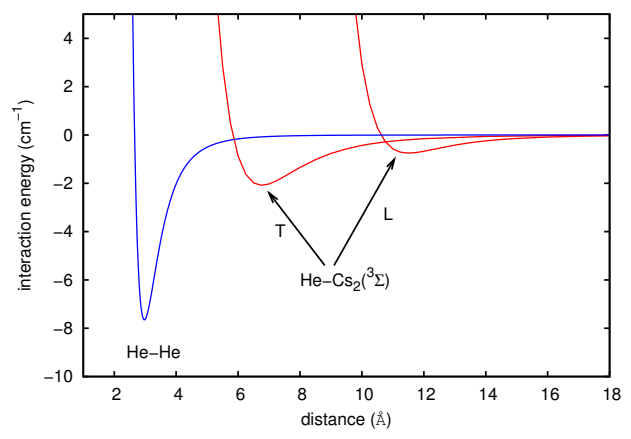

FIGURE 1. He-He dimer and He-Cs $2\left({ }^{3} \Sigma\right)$ trimer potential energy interactions. Linear (L) and T-shape (T) arrangements are depicted for the trimer. 
bound level at $\sim-0.10 \mathrm{~cm}^{-1}$ for the boson ${ }^{4} \mathrm{He}-\mathrm{Cs}_{2}\left({ }^{3} \Sigma\right)$ species. Figure 1 depicts the interaction energies for linear and perpendicular arrangements. Also in this figure we plot the He-He interaction[37] for comparison. As can be realized, the latter one presents its minimum of $\sim-8 \mathrm{~cm}^{-1}$ at a shorter distance of $\sim 3 \AA$. Taking into account that pure boson helium clusters quickly increase its binding energy with increasing size from $\sim 8 \times 10^{-4} \mathrm{~cm}^{-1}$ at $N=2$ to $\sim 5.50 \mathrm{~cm}^{-1}$ at $N=10[73]$, it is expected that the equilibrium configuration of ${ }^{4} \mathrm{He}_{N}-\mathrm{Cs}_{2}$ clusters should correspond to the cesium dimer attached to a (distorted) ${ }^{4} \mathrm{He}_{N}$ cluster.

Assuming the approach already mentioned, the PESs of $\mathrm{He}_{N}-\mathrm{Cs}_{2}$ clusters are analytically represented as a sum of $N \mathrm{Cs}_{2}$-He triatomic potentials[43] and $N(N-1) / 2 \mathrm{He}-\mathrm{He}$ pair interactions[37]

$$
V_{\mathrm{He}_{N}-\mathrm{Cs}_{2}}\left(\left\{\mathbf{R}_{k}\right\}_{k=1}^{N} ; r_{e q}\right)=\sum_{k=1}^{N} W\left(R_{k}, r_{e q}, \theta_{k}\right)+\sum_{k<l} V_{k l}\left(\left|\mathbf{R}_{k}-\mathbf{R}_{l}\right|\right),
$$

whith the bond-length of the impurity fixed at its equilibrium value, $r_{e q}=6.8 \AA$.

\section{Preliminary results}

PIMC calculations have been performed for different cluster sizes at a temperature of $1 \mathrm{~K}$ (it is actually hard to approach $0 \mathrm{~K}$ ). Thermalization was reached after $10^{6} \mathrm{MC}$ steps, and further $10^{6}$ steps were used for getting reliable statistics. For a cluster containing twelve boson helium atoms, Figure 2 shows the distribution of $\cos \gamma$ obtained within a classical description, i.e., $M=1$. As expected, the distribution is clearly restricted to a small region around $\cos \gamma=1$, that is, the He atoms remain gathered and, presumably, far from the cesium dimer in a quasi-perpendicular $\theta \simeq \pi / 2$ configuration. Of course, this conclusion needs to be further confirmed through $M>1$ quantum calculations.

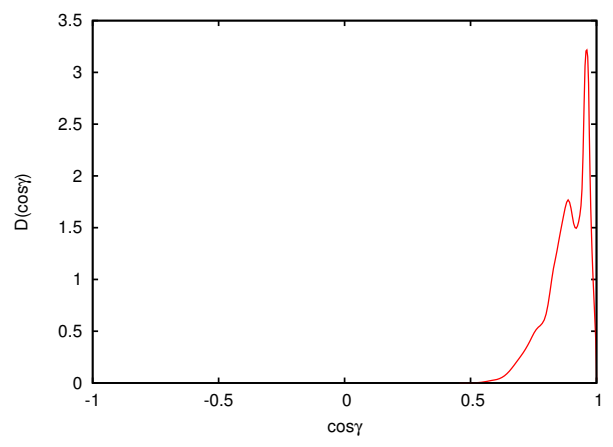

FIGURE 2. PIMC distribution of $\cos \gamma$

Also, QC calculations have been carried out on these boson species for even sizes from $N=2$ up to 12 . Within the $\mathrm{H}$ methodology, as above mentioned, it is necessary to avoid the strong He-He repulsion at short distances. Here, starting from the original He-He interaction[37], $V$, we adopt a truncated barrier scheme[27, 29], $V \rightarrow V \exp (-\alpha V)$. The $\alpha$ parameter was determined by matching of the energy to that obtained through variational calculations on the $\mathrm{He}_{2}-\mathrm{Cs}_{2}$ system $\left(-0.218 \mathrm{~cm}^{-1}\right)$, reaching a value of $2000 \mathrm{au}^{-1}$. A large basis set of type (14) with $n_{\max }=8$, $l_{\max }=11$, and $\left|m_{\max }\right|=1$ was considered. To obtain the $G_{n}$ radial functions, Eq. (15) was numerically solved using a Numerov procedure at 8 equally spaced configurations in $0 \leq \theta \leq \pi / 2.4096$ points were accounted for in the range of $2 \leq R \leq 200 \AA$. During the self-consistent optimization stage, the convergence thresholds for the energy and the module of its gradient were $10^{-6}$ and $10^{-5} \mathrm{~cm}^{-1}$, respectively. The initial orbitals were those states coming from the diagonalization of the triatomic $\mathrm{He}-\mathrm{Cs}_{2}$ cluster where the dimer rotation is neglected. We found just three bound orbitals, a $\Sigma$ state at an energy of $-0.107 \mathrm{~cm}^{-1}$, and two degenerate $\Pi$ states at $-0.064 \mathrm{~cm}^{-1}$, corresponding to $\Lambda= \pm 1$. Indeed, along the self-consistent procedure, virtual one-particle orbitals are also reached. Table 1 collects ground level energies, Eq. (12), of the different sized clusters depending on the $M$ number of orbitals accounted for. The oneparticle contribution of the first term in Eq. (12) is denoted as $\varepsilon$, while $J+K$ refers to the two-particle contribution of the second term. Also in this table, the energies of pure cluters[73] are shown. As can be realized, a single orbital is unable to describe clusters with $N \geq 6$ as their binding energies are lower than those corresponding to pure clusters, that is, the impurity would not be bounded to the helium atoms. This requirement is however fulfilled when $M=2$, 3 are considered. Note that two-particle contributions, coming from $\mathrm{He}-\mathrm{He}$ interactions, are dominant and increase 
TABLE 1. Energy $(E)$ and its breakdown, itemized in one $(\varepsilon)$ and two-particle $(J+K)$ contributions depending on the $N$ cluster size and the number $M$ of orbital accounted for. Last column collects DMC energies of pure clusters. Units are $\mathrm{cm}^{-1}$.

\begin{tabular}{r|ccc|ccc|ccc|c|}
\multicolumn{3}{c}{$M=1$} & \multicolumn{9}{c|}{$M=2$} \\
\hline$N$ & $E$ & $\varepsilon$ & $J+K$ & $E$ & $\varepsilon$ & $J+K$ & $E$ & $\varepsilon$ & $J+K$ & {$[73]$} \\
\hline 2 & -0.244 & -0.100 & -0.144 & -0.236 & -0.078 & -0.158 & & & & -0.0008 \\
4 & -0.66 & -0.18 & -0.48 & -0.79 & -0.08 & -0.71 & -0.84 & -0.06 & -0.78 & -0.37 \\
6 & -1.34 & -0.18 & -1.16 & -1.84 & +0.04 & -1.88 & -1.97 & +0.10 & -2.07 & -1.53 \\
8 & -2.35 & -0.10 & -2.25 & -3.47 & +0.26 & -3.73 & -3.77 & +0.32 & -4.09 & -3.29 \\
10 & -3.75 & +0.05 & -3.80 & -5.76 & +0.58 & -6.34 & -6.28 & +0.70 & -6.98 & -5.50 \\
12 & -5.58 & +0.27 & -5.85 & -8.76 & +1.00 & -9.76 & -9.65 & +1.21 & -10.86 & - \\
\hline
\end{tabular}

their relative importance with the cluster size. In turn, single-particle contributions decrease and, as consequence of a gradual filling of virtual orbitals, they even reach positive values. The picture emerging from these preliminary calculations is, according to PIMC results, that of the cesium dimer attached to a group of helium atoms, and not immersed in them. Calculations achieving convergency on the basis set and the number of orbitals, as well as different distributions of the He atoms respect to the dopant, and among them, are currently under progress.

\section{SUMMARY AND OUTLOOK}

We have outlined in this work a quantum chemistry-like methodology to study helium clusters doped with a diatomic impurity. It allows to perform spectral simulations depending on the polar/non polar character of the dopant as well as on the boson/fermion nature of the solvent. The main features exhibited by infrared spectra of carbonyl sulfide in helium nanodroplets are succesfully reproduced through our simulations, including the manifestation of microscopic superfluidity of boson environments.

Path-integral Monte Carlo methodology has been applied to the scenario in which the dopant is not embedded in the (boson) helium cluster but remains on its surface, as is the case of the cesium dimer in its ground triplet state. The situation is particularly challenging for central field-based approaches. However, preliminary Hartree calculations show that quantum-chemistry treatments are still suitable to study this kind of doped clusters in which the helium atoms no longer ocuppy a single quantum level.

There are several possible extensions of this methodology. Among them, one may consider clusters of para- $\mathrm{H}_{2}$ and orto- $\mathrm{H}_{2}$ as solvation environments where similar effects to those found in bosonic and fermionic helium clusters can be expected. Data obtained in spectroscopic studies of hydrogen clusters[74] and of different molecules embedded in them $[75,76,77]$ provide both a boost and a calibration tool for the present modeling.

Our main efforts for future work, however, address to developing a hierarchy of high-level ab initio methods to obtain accurate energies and wave-functions of bosonic, fermionic, and mixed doped helium clusters for ground and also low-lying excited states. This would allow to incorporate the interaction induced by the overall solvent orbital angular momentum-dopant rotation coupling[78]. Towards this goal, an efficient FCI treatment for small doped ${ }^{3} \mathrm{He}_{N}$ clusters have been already developed $[52,56]$. The results can serve as benchmarch to explore computationally less expensive methods as multiconfigurational self-consistent-field and multireference-CI treatments in order to tackle the study of larger clusters.

\section{ACKNOWLEDGMENTS}

This work has been partially supported by the MICINN (Spain) under projects FIS2007-62006 and CTQ200762898, and by CSIC-CAM (Spain) under grant CCG08-CSIC/ESP-3680. J. J. was supported by the Office of Basic Energy Sciences, Division of Chemical Sciences, Geosciences, and Biosciences, U.S. Department of Energy under Contract number DE-AC-02-06CH11357. The calculations presented here were performed at Centro de Calculo (IFF), CTI(CSIC), and CESGA (the SuperComputer Center of Galicia). 


\section{REFERENCES}

1. S. Goyal, D. L. Schutt, and G. Scoles, Phys. Rev. Lett. 69, 933 (1992).

2. M. Hartmann, R. E. Miller, J. Toennies, and A. F. Vilesov, Phys. Rev. Lett. 75, 1566 (1995).

3. C. Callegari, K. K. Lehmann, R. Schmied, and G. Scoles, J. Chem. Phys. 115, 10090 (2001).

4. J. P. Toennies, and A. F. Vilesov, Angew. Chem., Int. Ed. 43, 2622 (2004).

5. S. Grebenev, J. P. Toennies, and A. F. Vilesov, Science 279, 2083 (1998).

6. K. Nauta, and R. E. Miller, J. Chem. Phys. 115, 10138 (2001).

7. K. Nauta, and R. E. Miller, J. Chem. Phys. 117, 4846 (2002).

8. C. M. Lindsay, and R. E. Miller, J. Chem. Phys. 122, 104306 (2005).

9. J. Tang, and A. R. W. McKellar, J. Chem. Phys. 119, 754 (2003).

10. A. R. W. McKellar, J. Chem. Phys. 121, 6868 (2004).

11. S. Grebenev, M. Hartmann, M. Havenith, B. Sartakov, J. P. Toennies, and A. F. Vilesov, J. Chem. Phys. 112, 4485 (2000).

12. S. Baroni, and S. Moroni, in Quantum Monte Carlo Methods in Physics and Chemistry, edited by P. Nightingale, and C. J. Umrigar, NATO Series, Mathematical and Physical Sciences, Kluwer Academic, Boston, 1999, vol. 525.

13. F. Paesani, A. Viel, F. A. Gianturco, and K. B. Whaley, Phys. Rev. Lett. 90, 073401 (2003).

14. C. Di Paola, F. A. Gianturco, D. López-Durán, M. P. de Lara-Castells, G. Delgado-Barrio, P. Villarreal, and J. Jellinek, ChemPhysChem 6, 1348 (2005).

15. D. M. Ceperley, Rev. Mod. Phys. 67, 279 (1995).

16. Y. Kwon, D. M. Ceperley, and K. B. Whaley, J. Chem. Phys. 104, 2341 (1996).

17. Z. E. Zillich, F. Paesani, Y. Kwon, and K. B. Whaley, J. Chem. Phys. 123, 114301 (2005).

18. Z. Li, L. Wang, H. Ran, D. Xie, N. Blinov, P.-N. Roy, and H. Guo, J. Chem. Phys. 128, 22513 (2008).

19. R. P. de Tudela, M. Márquez-Mijares, T. González-Lezana, O. Roncero, S. Miret-Artés, G. Delgado-Barrio, and P. Villarreal, J. Chem. Phys. (submitted).

20. M. Barranco, R. Guardiola, S. Hernández, R. Mayol, J. Navarro, and M. Pí, J. Low. Temp. Phys. 142, 1 (2006).

21. J. D. Anderson, J. Chem. Phys. 63, 1499 (1975).

22. E. Sola, J. Casulleras, and J. Boronat, Phys. Rev. B 73, 092515 (2006).

23. P. Jungwirth, and A. I. Krylov, J. Chem. Phys. 115, 10214 (2001).

24. A. Heidenreich, U. Even, and J. Jortner, J. Chem. Phys. 115, 10175 (2001).

25. A. Heidenreich, and J. Jortner, J. Chem. Phys. 118, 10101 (2003).

26. P. M. Felker, J. Chem. Phys. 125, 184313 (2006).

27. D. López-Durán, M. P. de Lara-Castells, G. Delgado-Barrio, P. Villarreal, C. Di Paola, F. A. Gianturco, and J. Jellinek, Phys. Rev. Lett. 93, 053401 (2004).

28. D. López-Durán, M. P. de Lara-Castells, G. Delgado-Barrio, P. Villarreal, C. Di Paola, F. A. Gianturco, and J. Jellinek, J. Chem. Phys. 121, 2975 (2004).

29. M. P. de Lara-Castells, D. López-Durán, G. Delgado-Barrio, P. Villarreal, C. Di Paola, F. A. Gianturco, and J. Jellinek, Phys. Rev. A 71, 033203 (2005).

30. M. P. de Lara-Castells, R. Prosmiti, G. Delgado-Barrio, D. López-Durán, P. Villarreal, F. A. Gianturco, and J. Jellinek, Phys. Rev. A 74, 053201 (2006).

31. P. Villarreal, M. P. de Lara-Castells, R. Prosmiti, G. Delgado-Barrio, D. López-Durán, F. A. Gianturco, and J. Jellinek, Phys. Scr. 76, C96 (2007).

32. M. P. de Lara-Castells, R. Prosmiti, D. López-Durán, G. Delgado-Barrio, P. Villarreal, F. A. Gianturco, and J. Jellinek, Int. J. Quant. Chem. 107, 2902 (2007)

33. G. C. Schatz, R. B. Gerber, and M. A. Ratner, J. Chem. Phys. 88, 3709 (1988).

34. A. Valdés, R. Prosmiti, P. Villarreal, and G. Delgado-Barrio, J. Chem. Phys. 122, 044305 (2005).

35. R. Prosmiti, C. Cunha, P. Villarreal, and G. Delgado-Barrio, J. Chem. Phys. 116, 9249 (2002).

36. A. Valdés, R. Prosmiti, P. Villarreal, and G. Delgado-Barrio, Mol. Phys. 102, 2277 (2004).

37. R. A. Aziz, and M. J. Slaman, J. Chem. Phys. 94, 8047 (1991).

38. R. Prosmiti, C. Cunha, P. Villarreal, and G. Delgado-Barrio, J. Chem. Phys. 117, 7017 (2002).

39. A. Valdés, R. Prosmiti, P. Villarreal, and G. Delgado-Barrio, J. Chem. Phys. 125, 014313 (2006).

40. W. E. Ernst, R. Huber, S. Jiang, R. Beuc, M. Movre, and G. Pichler, J. Chem. Phys. 124, 024313 (2006).

41. D. Li, F. Xie, L. Li, S. Magnier, V. B. Sovkov, and V. S. Ivanov, Chem. Phys. Lett. 441, 39 (2007).

42. D. DeMille, S. Sainis, J. Sage, T. Bergeman, S. Kotochigova, and E. Tiesinga, Phys. Rev. Lett. 100, 043202 (2008).

43. R. Prosmiti, G. Delgado-Barrio, P. Villarreal, E. Yurtsever, E. Coccia, and F. A. Gianturco, J. Phys. Chem. A (in press).

44. S. Bovino, E. Coccia, E. Bodo, D. López-Durán, and F. A. Gianturco, J. Chem. Phys. 130, 224903 (2009).

45. P. Villarreal, O. Roncero, and G. Delgado-Barrio, J. Chem. Phys. 101, 2217 (1994).

46. O. Roncero, R. Pérez-de Tudela, M. P. de Lara-Castells, R. Prosmiti, G. Delgado-Barrio, and P. Villarreal, Int. J. Quant. Chem. 107, 2756 (2007).

47. O. Roncero, M. P. de Lara-Castells, G. Delgado-Barrio, P. Villarreal, T. Stoecklin, A. Voronin, and J. C. Rayez, J. Chem. Phys. 128, 164313 (2008).

48. G. Maroulis, J. Chem. Phys. 118, 2673 (2003). 
49. P. Barletta, A. Fabrocini, A. Kievsky, J. Navarro, and A. Polls, Phys. Rev. A 68, 053205 (2003).

50. J. Fernández-Rico, "Direct minimization in Hartree-Fock Methods," in Self-Consistent Field, Theory and Applications, edited by R. Carbo, and M. Klobukowski, Physical and Theoretical Chemistry (Elsevier), 1990, vol. 70.

51. J. Fernández-Rico, J. M. G. de la Vega, M. Paniagua, and J. I. Fernández-Alonso, J. Chem. Phys. 79, 4407 (1983)

52. M. P. de Lara-Castells, G. Delgado-Barrio, P. Villarreal, and A. O. Mitrushchenkov, J. Chem. Phys. 125, 221101 (2006).

53. G. L. Sleijpen, and H. A. Van der Vorst, SIAM J. Matrix Anal. Appl. 17(2), 401 (1996).

54. E. R. Davidson, J. Comput. Phys. 17, 87 (1975).

55. M. P. de Lara-Castells, A. O. Mitrushchenkov, G. Delgado-Barrio, and P. Villarreal, Few-Body Syst 45, 233 (2009).

56. M. P. de Lara-Castells, P. Villarreal, G. Delgado-Barrio, and A. O. Mitrushchenkov, J. Chem. Phys. 131, 194101 (2009).

57. P. Borrmann, Comp. Mat. Sci. 2, 593 (1994).

58. M. F. Herman, E. J. Bruskin, and B. J. Berne, J. Chem. Phys. 76, 5150 (1982).

59. K. R. Glaesemann, and L. E. Fried, J. Chem. Phys. 116, 5951 (2002).

60. N. Metropolis, A. W. Rosenbluth, M. N. Rosenbluth, A. H. Teller, and E. Teller, J. Chem. Phys. 21, 1087 (1953).

61. E. B. Wilson, Jr., J. C. Decius, and P. C. Cross, Molecular Vibrations: The Theory of Infrared and Raman Vibrational Spectra, McGraw-Hill Book Company, Inc. (Reprinted by Dover Publications in 1980), New York, 1955.

62. B. Miguel, A. Bastida, J. Zúñiga, A. Requena, and N. Halberstadt, Faraday Discuss. 118, 257 (2001).

63. M. I. Hernández, T. González-Lezana, A. Buchachenko, R. Prosmiti, M. P. de Lara-Castells, G. Delgado-Barrio, and P. Villarreal, "Photo-predissociation of the ${ }^{4} \mathrm{He}^{79} \mathrm{Br}_{2}$ complex: From empirical to ab initio potential energy surfaces," in Recent research developments in Chemical Physics, Transworld Research Network, Kerala, India, 2003, vol. 4, p. 1.

64. P. Villarreal, A. Varadé, and G. Delgado-Barrio, J. Chem. Phys. 90, 2684 (1989).

65. O. Roncero, G. Delgado-Barrio, M. I. Hernández, J. Campos-Martínez, and P. Villarreal, Chem. Phys. Lett. 246,187 (1995).

66. M. I. Hernández, A. García-Vela, J. Campos-Martínez, O. Roncero, P. Villarreal, and G. Delgado-Barrio, Comput. Phys. Commun. 145, 97 (2002).

67. J. A. Beswick, and G. Delgado-Barrio, J. Chem. Phys. 73, 3653 (1980).

68. G. Maroulis, and C. Makris, Mol. Phys. 91, 333 (1997).

69. J. P. Toennies, A. F. Vilesov, and K. B. Whaley, Phys. Today 54, 31 (2001).

70. M. Foucrault, P. Millie, and J. P. Daudey, J. Chem. Phys. 96, 1257 (1992).

71. F. Xie, V. B. Sovkov, A. M. Lyra, D. Li, S. Ingram, J. Bai, V. S. Ivanov, S. Magnier, and L. Li, J. Chem. Phys. 130, 051102 (2009).

72. R. B. Ross, J. M. Powers, T. Atashroo, W. C. Ermler, L. A. LaJohn, and P. A. Christiansen, J. Chem. Phys. 93, 6654 (1990).

73. M. Lewerenz, J. Chem. Phys. 106, 4596 (1997).

74. G. Tejada, J. M. Fernández, S. Montero, D. Blume, and J. P. Toennies, Phys. Rev. Lett. 92, 223401 (2004).

75. S. Grebenev, B. Sartakov, J. P. Toennies, and A. F. Vilesov, Science 289, 1532 (2000).

76. J. Tang, and A. R. W. McKellar, J. Chem. Phys. 121, 3087 (2004).

77. S. Moroni, M. Botti, S. De Palo, and A. R. W. McKellar, J. Chem. Phys. 122, 094314 (2005).

78. M. P. de Lara-Castells, A. Mitrushenkov, P. Palmieri, F. L. Quére, C. Leonard, and P. Rosmus, Mol. Phys. 98, 1713-1727 (2000). 